\title{
Des prisonnières en guerre : le cas des femmes condamnées à mort au sortir de l'Occupation
}

Prisoners of war: the case of women condemned to death in the aftermath of the occupation

Fabien Lostec

\section{(2) OpenEdition}

Journals

\section{Édition électronique}

URL : http://journals.openedition.org/abpo/3671

DOI : 10.4000/abpo.3671

ISBN : 978-2-7535-6497-8

ISSN : 2108-6443

Éditeur

Presses universitaires de Rennes

\section{Édition imprimée}

Date de publication : 30 juin 2017

Pagination : 121-143

ISBN : 978-2-7535-6495-4

ISSN : 0399-0826

Référence électronique

Fabien Lostec, «Des prisonnières en guerre : le cas des femmes condamnées à mort au sortir de I'Occupation", Annales de Bretagne et des Pays de l'Ouest [En ligne], 124-2 | 2017, mis en ligne le 17 juillet 2019, consulté le 29 janvier 2021. URL : http://journals.openedition.org/abpo/3671 ; DOI : https://doi.org/10.4000/abpo.3671 


\title{
Des prisonnières en guerre : le cas des femmes condamnées à mort au sortir de l'Occupation ${ }^{1}$
}

\author{
Fabien LOSTEC \\ Doctorant TEMPORA EA7468 - université Rennes 2
}

Dans ses mémoires, Mathilde Carré, alias " la chatte ", ancienne espionne au service des nazis, évoque la journée du 13 juin 1949, au cours de laquelle elle fit son " entrée dans la Centrale de Rennes ", quelques mois après la sentence la condamnant à la peine capitale ${ }^{2}$. " On nous aligna dans la partie réservée où passent toutes les prisonnières à leur arrivée et au départ précise-t-elle, une espèce d'isoloir, long couloir bordé de cellules et se terminant par le "gnouf" ou "mitard". " C'est dans la prison bretonne, en effet, que la plus grande partie des femmes condamnées à mort au titre de l'épuration furent emprisonnées à compter de 1944.

Depuis une vingtaine d'années, les historiens ont décentré leur regard afin d'analyser cette épuration, non plus comme l'ultime avatar de la Seconde Guerre mondiale, mais comme une forme à part entière de la démobilisation de la société. Davantage que l'épuration en France, c'est la société française en épuration qui est étudiée. Loin d'être anodin, ce changement de prisme permet de mieux connaître le vécu et le perçu de milliers de Français "d'en bas " face à l'épuration ${ }^{3}$. Au sein de ce phénomène social d'ampleur, une catégorie de population - les femmes - et un lieu - la prison - occupent une place centrale ${ }^{4}$. Pourtant, à l'exception

1. J'adresse mes vifs remerciements à Yann Lagadec, qui est à l'origine de cet article et l'a suivi jusqu'à sa publication, ainsi qu'à Marc Bergère, pour sa disponibilité et ses remarques, qui ont beaucoup apporté à ce travail. Merci enfin à Thierry Lhote, Étienne et Jean-Baptiste Mathieu.

2. CARRÉ, Lily, On m'appelait la chatte, Paris, Albin Michel, 1975, p. 271, juin 1949.

3. Sur ces déplacements de regard, voir notamment les travaux de BERGĖRE, Marc, Une société en épuration. Épuration vécue et perçue en Maine-et-Loire. De la Libération au début des années 50, Rennes, PUR, 2004.

4. Sur l'épuration judiciaire ou extrajudiciaire des femmes, voir LECLERC, Françoise, WEINDLING, Michèle, "La répression des femmes coupables de collaboration ", CLIO, Histoire, Femmes et Sociétés, $n^{\circ}$ 1, 1995, p. 129-150, ou "Des femmes devant les cours de justice", 
de l'internement administratif, les historiens ont rarement fait dialoguer ces deux champs de recherche, plaçant les femmes privées de liberté à la Libération dans une sorte d'impensé scientifique ${ }^{5}$. Si elles ne peuvent être considérées comme des prisonnières de guerre, elles n'en demeurent pas moins des prisonnières en guerre ${ }^{6}$. En effet, pendant que l'épuration s'ancre dans un climat de guerre civile, le conflit mondial, lui, se poursuit sur d'autres fronts ${ }^{7}$.

Cet angle mort historiographique est d'autant plus surprenant que les prisons sont un véritable enjeu durant une période marquée par l'explosion de la population carcérale. Toutes catégories confondues, le nombre de prisonniers culmine à 70000 en 1945, soit plus de quatre fois celui d'avantguerre $^{8}$. La diminution est ensuite régulière mais les prisons demeurent longtemps surchargées : elles abritent encore 62000 personnes en 1946, 57000 en 1948 et environ 36000 en 1950. Traditionnellement sous-représentées dans la population carcérale (environ $8 \%$ dans les années 1930 et les années 1950), les femmes forment, à la Libération une part bien plus

dans KANDEL, Liliane (dir.), Féminismes et nazisme, Paris, Odile Jacob, 2004 [1997], p. 74-85; CAPDEVILA, Luc, Les Bretons au lendemain de l'Occupation. Imaginaire et comportement d'une sortie de guerre 1944-1945, Rennes, PUR, 1999; VIRGILI, Fabrice, La France " virile ». Des femmes tondues à la Libération, Paris, Payot, 2004 [2000]; BERGÈRE, Marc, Une société en épuration..., op. cit.; ou encore Simonin, Anne, Le Déshonneur dans la République. Une histoire de l'indignité 1791-1958, Paris, Grasset, 2008.

5. Les études sur l'emprisonnement des collaborateurs à la Libération n'évoquent pas les femmes. Voir par exemple Vergez-Chaignon, Bénédicte, Vichy en prison. Les épurés à Fresnes après la Libération, Paris, Gallimard, 2006, ou VIMONT, Jean-Claude, " Les fers des condamnés à mort. Détention carcérale et instrumentalisation politique durant l'Épuration en France ", dans Porret, Michel, Fontana, Vincent, MAugue, Ludovic (dir.), Bois, fers et papiers de justice. Histoire matérielle du droit de punir, Genève, L'Équinoxe, 2012, p. 299312. Si les collaboratrices emprisonnées à la Libération furent très rarement étudiées, ce n'est pas le cas des prisonnières sous l'Occupation. Voir JALADIEU, Corinne, La prison politique sous Vichy. L'exemple des centrales d'Eysses et de Rennes, Paris, L'Harmattan, 2007. De manière plus large, sur la prison sous l'Occupation, se reporter à PÉDRON, Pierre, La prison sous Vichy, Paris, Les éditions de l'Atelier, 1993.

6. Les prisonnières en guerre ne doivent pas être confondues avec les combattantes rattachées aux différentes armées. Comme le prévoit la convention de Genève de 1929, une fois capturées, celles-ci bénéficient du statut de prisonnières de guerre (CAPDEVILA, Luc, RouQuet, François, VIRGILI, Fabrice, Voldman, Danièle, Hommes et femmes dans la France en guerre (1914-1945), Paris, Payot, 2003, p. 284-287). En cela, les prisonnières en guerre qui nous intéresseront ici illustrent tout l'intérêt que peuvent avoir ces statuts de l'entre-deux, entre mondes civil et militaire, à l'instar de ce qu'a pu montrer, dans ce même numéro des $A B P O$, l'article d'Hervé Le Goff sur la captivité au temps de la Ligue en Bretagne.

7. Le concept de guerre civile est toutefois à manier avec précaution en ce qui concerne les années 1944-1945. Celle-ci correspond moins à une réalité qu'à un ressenti. Sur les débats autour de cette question, se reporter notamment à WIEVIORKA, Olivier, " Guerre civile à la française? Le cas des années sombres (1940-1945) ", Vingtième siècle. Revue d'histoire, $\mathrm{n}^{\circ} 85,2005 / 1$, p. 5-19.

8. Vergez-Chaignon, Bénédicte, Vichy en prison..., op. cit., p. 12. PÉDron, Pierre, La prison sous Vichy, op. cit., p. 199, précise quant à lui que le pic est uniquement atteint en 1946 avec un peu plus de 60000 prisonniers. Voir également BARRE, Marie-Danièle, " 130 années de statistique pénitentiaire en France ", Déviance et société, no 2, vol. 10, 1986, p. 115. 
importante des prisonniers : elles représentent $18 \%$ d'entre eux à l'échelle nationale et, par exemple, $20 \%$ de ceux de Fresnes ou $34 \%$ des suspects de collaboration internés dans le camp de Châteaubriant ${ }^{9}$.

À cette explosion s'adjoint une grande " confusion pénitentiaire " du fait de l'extrême diversité des statuts des individus enfermés ${ }^{10}$. Interroger l'emprisonnement des femmes - ses commanditaires, ses lieux, ses conditions, ses objectifs et ses issues -, c'est donc mieux saisir la polysémie de ce terme ainsi que les différentes scansions qui rythment la sortie de guerre. Car, même s'il convient d'insister sur l'extrême fluidité et la coexistence des catégories, leur évolution épouse la chronologie du progressif, et parfois difficile, rétablissement de l'autorité républicaine ${ }^{11}$. Dans un premier temps, qui court du débarquement de Normandie jusqu'à la libération effective du territoire, l'épuration populaire précède l'épuration légale. Exceptionnellement, durant cette phase insurrectionnelle où ses préoccupations sont ailleurs, la Résistance enferme des femmes que l'on peut alors considérer comme captives. Une seconde séquence correspond aux premières journées de la Libération. Pendant une brève période d'anomie entre le départ des Allemands et l'installation des nouveaux pouvoirs, l'attitude de ces derniers vis-à-vis des châtiments exercés par les communautés à l'encontre des collaborateurs est très ambiguë, plaçant l'épuration populaire aux frontières de la légalité. À cet instant, le nombre de captives est à son acmé. Il faut plusieurs jours avant que la légitimité de droit ne reprenne l'ascendant sur une légitimité de fait détenue par la Résistance locale. Pour ce faire, les pouvoirs publics internent administrativement les individus suspectés de collaboration ${ }^{12}$. Première forme d'épuration légale, cet internement cherche à répondre à l'afflux croissant des arrestations

9. Vergez-Chaignon, Bénédicte, Vichy en prison..., op. cit., p. 16, et Peschanski, Denis, "L'internement des femmes dans la France des années noires ", dans FualKow, Jacques (dir.), Les femmes dans les années quarante. Juives et non-Juives, souffrances et résistance, Paris, Les éditions de Paris, 2004, p. 95-112. Parmi toutes les formes d'enfermement, l'internement administratif semble être celle où la part des femmes est la plus forte. Notons toutefois que la part des femmes parmi les prisonniers avait déjà connu une augmentation significative sous l'Occupation du fait de l'incarcération des résistantes. Cela démontre que les femmes se sont fortement engagées durant l'Occupation, dans un camp comme dans l'autre.

10. Selon les mots de PÉdron, Pierre, La prison sous Vichy, op. cit., p. 195.

11. Cette chronologie s'inspire grandement de celle dressée par BERGÈRE, Marc, "Pratiques et régulations des violences intracommunautaires dans la société française en épuration ", dans Follain, Antoine, LEMESLE, Bruno, NASSIET, Michel, PIERRE, Éric, QUINCYLEFEBVRE, Pascale (dir.), La violence et le judiciaire. Discours, perceptions, pratiques, Rennes, PUR, 2008, p. 326.

12. L'internement administratif ayant fait l'objet de solides études, nous faisons le choix ne pas le placer au cœur de ce texte pour se concentrer sur d'autres formes d'enfermement, bien moins connues. Le lecteur désireux d'en savoir plus sur l'internement pourra se reporter à PESCHANSKI, Denis, La France des camps. L'internement, 1938-1946, Paris, Gallimard, 2002, BERGÈRE, Marc, Une société en épuration..., op. cit., p. 65-74 et 169180, ou, plus récemment, DuguET, Laurent, Incarcérer les collaborateurs. Dans les camps de la Libération, 1944-1945, Paris, Vendémiaire, 2015. 
tout en diminuant le nombre de captifs. Jusqu'au milieu de l'année 1945, la population internée continue d'augmenter, avant d'entamer sa décrue. Après examen par la commission départementale de vérification des internements qui avaient été décidés par le préfet, certaines femmes internées sont versées dans la catégorie des détenues. En vertu d'un mandat du juge d'instruction, elles sont emprisonnées de manière provisoire et attendent leur jugement. Et ce n'est qu'après avoir été condamnées par les juridictions de l'épuration légale que des femmes sont incarcérées. Pleinement rétabli, l'État de droit demeure cependant fragile. Désormais centralisées et officialisées, des prisons sont attaquées par la Résistance et/ou les populations. L'épuration populaire cherche ainsi à corriger une épuration légale considérée comme trop timorée ${ }^{13}$.

Pour sortir ces femmes des " ombres de l'histoire", nous avons choisi d'examiner celles qui sont le plus lourdement sanctionnées à la Libération et qui demeurent en quelque sorte les dernières prisonnières de la Seconde Guerre mondiale en France : les condamnées à mort ${ }^{14}$. Au-delà d'une plongée dans le monde de l'engagement au service de l'Allemagne, fréquemment considéré comme exclusivement masculin, leur étude donne accès à l'univers carcéral féminin, d'autant que plusieurs d'entre elles sont successivement captives, internées, détenues et incarcérées. Leur recensement exhaustif, réalisé à l'échelle nationale, nous permet de constater que, sans être négligeable, la part des femmes exécutées demeure faible et principalement le fait des tribunaux militaires, plus ou moins légaux, qui siègent avant l'installation des cours de justice par le Gouvernement provisoire de la République française (GPRF) en octobre 1944. La grâce est alors accordée à une grande majorité d'entre elles, permettant de commuer la peine capitale en une peine de travaux forcés à perpétuité et de les incarcérer. Mais le contraste entre les peines prononcées et les peines subies est important. Ces femmes recouvrent la liberté au plus tard à la fin des années 1950.

La majorité d'entre elles (en l'état de nos recherches, 142 des 192 condamnées à mort graciées dont nous connaissons le parcours carcéral, soit près des trois-quarts) ont séjourné, à un moment ou un autre, à la maison centrale de Rennes ${ }^{15}$. Construit dans la seconde moitié du XIX ${ }^{\mathrm{e}}$ siècle, il s'agit du premier établissement pénitentiaire français destiné à accueillir uniquement des femmes. De grandes figures de la criminalité féminine comme Thérèse Humbert, les sœurs Papin ou encore Violette Nozière, y ont notamment séjourné dans l'entre-deux-guerres. L'Occupation achevée, des prisonnières en chassent d'autres et les collaboratrices remplacent

13. On constate notamment une réactivation des violences populaires au printemps et à l'été 1945, lors du rapatriement des déportés.

14. Perrot, Michelle, Les ombres de l'histoire. Crime et châtiment au XIXe siècle, Paris, Flammarion, 2001. L'étude des femmes condamnées à mort pour faits de collaboration est au cœur de notre thèse sur Le collaborationnisme des femmes au miroir de l'épuration judiciaire en France à la Libération, réalisée sous la direction de Marc Bergère.

15. Seules les juridictions civiles ont ici été prises en compte. Les 192 femmes dont il est ici question ont donc toutes été condamnées à mort par des cours de justice. 
les résistantes qui peuplaient les cellules quelques semaines auparavant. La prison dispose, avec les dossiers des femmes incarcérées conservés aux Archives départementales d'Ille-et-Vilaine, d'archives riches et originales. Ceux-ci complètent utilement les dossiers de procédure et de recours en grâce $^{16}$. De cette façon, nous disposons d'une vue d'ensemble sur le parcours de cette "poignée de [femmes] misérables", de leur action sous l'Occupation à leur sortie de prison ${ }^{17}$.

En puisant dans ce matériau, nous tenterons d'abord de cerner la réalité, souvent brouillée, de l'emprisonnement à la Libération avant, dans un second temps, d'étudier le quotidien des femmes incarcérées.

\section{Être prisonnière à la Libération}

Instrument aux mains des Allemands sous l'Occupation, la prison ne tarde pas à devenir un lieu "stratégique " à la Libération : elle doit absorber les milliers de suspects de collaboration arrêtés dès les premières heures de la délivrance et parfois même avant, de manière clandestine. À cet instant, l'emprisonnement est marqué par l'immédiateté des actes et l'incertitude des acteurs, qu'il s'agisse des femmes épurées ou des épurateurs. Puis, à mesure du rétablissement de l'État de droit, le doute ne concerne plus que l'issue de procédures définies, connues et légales. À sa manière, l'emprisonnement témoigne donc de l'agitation puis de la progressive normalisation de la situation qui caractérisent la sortie de guerre.

\section{Dans le tumulte de la Libération : des femmes captives}

À la suite du débarquement de Normandie, l'épuration s'amplifie, dans une phase insurrectionnelle et de transition politique. Des résistants traquent les collaboratrices considérées comme dangereuses. Pour elles, le temps de captivité est empreint de violences et relativement bref, en raison de l'absence d'enquête et de l'inconsistance des interrogatoires. Il précède un jugement tout aussi rapide, rendu par un tribunal extralégal, qui ne respecte pas les règles les plus élémentaires du droit. Tout se passe

16. Les dossiers des prisonnières de la maison centrale de Rennes sont conservés sous la cote 278 W aux Archives départementales d'Ille-et-Vilaine; les dossiers de procédure se trouvent quant à eux dans les différents services des Archives des départements dans lesquels les femmes ont été condamnées à mort - ou dans le département qui abrite le siège de la cour d'appel dont dépendait la cour de justice; les dossiers de procédure des femmes condamnées par les tribunaux militaires se trouvent au Dépôt central des archives de la justice militaire, situé au Blanc; enfin, les dossiers de recours en grâce ont été consultés au Centre des Archives contemporaines de Fontainebleau. Ils sont aujourd'hui conservés aux Archives nationales, à Pierrefitte-sur-Seine.

17. Pour reprendre et adapter les mots prononcés par le général de Gaulle lors de son discours radiodiffusé du 14 octobre 1944, titre éponyme de la principale synthèse disponible sur l'épuration à l'heure actuelle : BARUCH, Marc-Olivier (dir.), Une poignée de misérables : l'épuration de la société française après la Seconde Guerre mondiale, Paris, Fayard, 2003. 
comme si, ainsi que l'écrit Marc Bergère, la Résistance « ressentait le besoin de légitimer l'usage de la violence par une parodie de procédure ${ }^{18}$ ".

Ces femmes sont souvent condamnées à la peine capitale et exécutées. Par exemple, au début du mois de juillet 1944, presque deux mois avant la libération de Mende, le lieutenant-colonel Peytavin, commandant la subdivision militaire FFI de la Lozère, donne ordre au capitaine Ameil de rechercher les agents à la solde des Allemands chargés d'espionner le maquis ${ }^{19}$. Toute personne étrangère à la région est considérée comme suspecte. Accompagné d'une vingtaine de résistants, Ameil emprisonne plus de deux cents suspects, dont une partie dans la grange d'une ferme située dans le nord du département. Dans cette grange gardée jour et nuit débute alors une vie collective en vase clos, où règnent la promiscuité et le manque d'hygiène ${ }^{20}$. Parmi les captifs, 33 hommes et 12 femmes sont déférés devant " le conseil de guerre de l'état-major du maquis de la Haute-Lozère " qui ne prononce qu'une seule sentence, la mort, immédiatement exécutoire. À la Libération, les familles des condamnés à mort demandent l'exhumation des corps et la restitution de l'argent prélevé au moment des arrestations. Une information judiciaire est ouverte et de nombreux témoins se plaignent des sévices exercés par les résistants. Certains font état des cris de souffrance entendus et " des traces de brutalités extrêmes " qu'ils ont vues sur les corps des prisonniers revenant des interrogatoires. D'autres révèlent les tortures auxquelles ils ont été soumis pour obtenir des aveux de culpabilité. Interrogés, les résistants, désormais inculpés, reconnaissent que " quelques " captifs ont été questionnés complètement nus « pour leur ôter leur assurance " et qu'ils ont donné des gifles, des coups de poing ou même effectué des " passages à tabac ". Par contre, ils nient avoir brûlé les poils pubiens d'une prisonnière et lui avoir introduit une bougie dans le vagin à coups de pied comme l'affirment des témoins. Seul un des résistants inculpés reconnaît avoir "bien fait le simulacre " de lui brûler les poils du sexe avec une allumette ${ }^{21}$. À l'interface d'une logique de guerre et de l'épuration stricto sensu, ces violences résistantes répondent à la multiplication des exactions opérées par les Allemands ou les ultras de la collaboration dans les régions à forte présence de maquis. Ainsi, si cet exemple demeure sans doute exceptionnel par la précocité et l'ampleur des arrestations, la Lozère n'étant libérée qu'à la fin du mois d'août 1944, il n'en demeure pas

18. BERGÈre, Marc, "Pratiques et régulations des violences intracommunautaires... ", art. cit., p. 327.

19. La ville de Mende est libérée le 21 août 1944.

20. CoRdesse, Henri, La libération en Lozère 1944-1945, Montpellier, Presses du Languedoc, 1977, p. 78.

21. Dépôt central des Archives de la justice militaire, minute de l'ordonnance de non-lieu rendu par le tribunal militaire permanent de Marseille le 25 novembre 1953 à l'endroit des résistants qui étaient accusés d'assassinats, de coups et blessures volontaires, de vols et d'arrestations arbitraires. Le tribunal estime qu'il n'y a pas lieu à poursuites notamment car " il semble que le Conseil de Guerre des maquis de la Haute-Lozère ait commis [son action] dans l'esprit de servir la cause de la libération définitive du territoire français " et non " pour des motifs d'intérêt personnel de lucre ou de vengeance". 
moins que des résistants ont pu s'encombrer de prisonniers et de prisonnières pendant plusieurs semaines. Comme le souligne Henri Cordesse, résistant et préfet de la Lozère à la Libération, cet emprisonnement a été réalisé " avec les moyens du bord, c'est-à-dire dans des conditions matérielles risquées pour les FFI et pénibles pour les suspects ${ }^{22}$ ".

Une fois la libération effective, la captivité des collaboratrices est rarement clandestine. Les lieux d'emprisonnement se multiplient, à la fois ostensibles et improvisés : les commissariats, les mairies mais aussi les écoles, laissées vacantes par leurs hôtes habituels du fait des vacances scolaires, ou encore les maisons de particuliers, sont utilisés à cet effet ${ }^{23}$. Parallèlement, des femmes suspectées de collaboration sont appréhendées et épurées. Le temps de l'arrestation se confond alors avec celui de la captivité $^{24}$. À Monterfil, une petite commune située à quelques kilomètres à l'ouest de Rennes, trois femmes qui travaillaient sous l'Occupation dans un camp occupé par une unité allemande des transmissions, sont arrêtées le 4 août 1944 par une douzaine de jeunes résistants. Ils les accusent d'avoir couché avec l'occupant et dénoncé certains d'entre eux. Avant d'être exécutées dans un bois alentour, les trois prisonnières sont frappées et tondues; dénudées, elles sont recouvertes de croix gammées et attachées durant plusieurs heures au cœur du village ${ }^{25}$. On peut y voir, à l'instar de Fabrice Virgili, la volonté des résistants de se réapproprier le corps des femmes et de réaffirmer une virilité mise à mal par la défaite de 1940 et quatre années de domination allemande ${ }^{26}$. Surtout, au cœur de l'espace public, le corps des prisonnières devient un enjeu et la captivité un temps partagé avec les populations locales. Car si celles-ci se sont dédouanées sur les résistants quelques années après les faits à l'évocation de cette épuration populaire, les violences ont bel et bien été effectuées dans un cadre communautaire.

22. CoRdesse, Henri, La libération en Lozère..., op. cit., p. 78. Notons que les tribunaux extralégaux se multiplient suite au départ des Allemands, à l'image de celui de Pamiers (Ariège) étudié par Pierre Laborie. Ici, entre le 18 - date de libération de la ville - et le 31 août 1944, 200 arrestations sont ordonnées. Tandis que 48 personnes - dont 16 femmes - sont libérées le 29 août, 42 individus au moins - dont huit femmes et sept militaires allemands - sont fusillés publiquement après avoir été jugés par un " tribunal du peuple ". Voir LABORIE, Pierre, "L'Épuration en Ariège ", dans LABORIE, Pierre, Les Français des années troubles. De la guerre d'Espagne à la Libération, Paris, Le Seuil, 2003 [2001], p. 227-243.

23. Pensons également à l'Institut dentaire parisien où, au minimum, 38 personnes, dont neuf femmes, furent exécutées après des jugements sommaires (BERLIERE, Jean-Marc et LiaIGRE, Franck, Ainsi finissent les salauds. Séquestrations et exécutions clandestines dans Paris libéré, Paris, Robert Laffont, 2012).

24. VIRGILI, Fabrice, "La tondue de la Libération. La traîtresse ", dans GAUvARD, Claude et al. (dir.), Présumées coupables, Paris, L'iconoclaste/Archives nationales, 2016, p. 241.

25. Arch. dép. d'Ille-et-Vilaine, 1044 W 169, dossier du Service régional de police judiciaire $n^{\circ} 14618$ et Dépôt central des Archives de la justice militaire, dossiers de non-lieu $\mathrm{n}^{\circ} 78$ et $\mathrm{n}^{\circ} 87$ du tribunal militaire de Rennes, respectivement datés du 3 et du 30 juillet 1945. Tous ces dossiers concernent Louis Oberthür, l'un des résistants ayant opéré l'épuration à Monterfil.

26. VIRGILI, Fabrice, La France " virile »..., op. cit. 
La population ne demeure pas inactive et participe à ces scènes de violence en criant, en insultant, voire en frappant les collaboratrices.

Ces captivités hors les murs, propices à la vengeance, posent logiquement la question de la réaction des policiers et des gendarmes, garants de l'ordre public. De fait, durant cette phase d'anomie, l'épuration des femmes suspectées de collaboration est régulièrement avalisée par les pouvoirs locaux ${ }^{27}$. Par leur non-intervention, leur accord, voire leur participation en tant qu'organisateurs, ils donnent à cette forme d'épuration un caractère semi-officiel. C'est d'ailleurs pour cela que la captivité exercée dans les premiers jours de la délivrance, illégale du point de vue du droit, n'a bien souvent pas été ressentie comme telle par les populations. Parfois même, elle n'est pas considérée comme une manifestation de désordre, mais comme un mode de régulation sociale au même titre que la détention ${ }^{28}$.

\section{Vers la détention : des prisonnières dans les mains des autorités républicaines}

L'emprisonnement ne se confond pas systématiquement avec l'épuration, qui ne saurait elle-même se résumer à une succession de scènes sauvages ou de jugements qui mènent aveuglément à l'exécution, sans prise en compte de la parole des captives.

Le 7 septembre 1944, soit quatre jours avant la libération de Dijon, Georgette Guéritey est jugée par un conseil de guerre FFI. Il lui est reproché d'avoir été la maîtresse d'un feldwebel allemand, avec lequel elle a eu un enfant, alors que son mari était prisonnier de guerre, et d'avoir dénoncé des habitants de son village aux Allemands ${ }^{29}$. Par ailleurs, elle a caché son amant sous le plancher de sa chambre à coucher après la Libération; l'homme se disait déserteur mais, retrouvé en possession d'un ordre de mission en date du mois de juillet 1944, il fut considéré par les résistants comme un espion obtenant des renseignements par l'intermédiaire de sa maîtresse. D'après le président de ce tribunal, l'inculpée a été interrogée pendant plus d'une heure et a reconnu les faits; elle est donc condamnée à la peine capitale pour intelligence avec l'ennemi. Toutefois, le conseil de guerre décide de surseoir à son exécution et de la maintenir en " garde à

27. Sur ce point, voir CAPDEVILA, Luc, Les Bretons au lendemain de l'Occupation..., op. cit., p. 237-260 notamment.

28. Cette violence, située à la frontière de l'infrajustice, voire de la parajustice, a été particulièrement étudiée pour l'époque moderne par GARNOT, Benoît, " Justice, infrajustice, parajustice et extra-justice dans la France d'Ancien Régime ", Crime, Histoire et Sociétés, $\mathrm{n}^{\circ}$ 1, vol. 4, 2000, p. 114. Pour la Libération, se reporter à BERGÈre, Marc, "Pratiques et régulations des violences intracommunautaires..." ", art. cit., p. 323-332.

29. Arch. dép. de la Côte-d'Or, 29 U 2/14, dossier de la cour de justice de la Côte-d'Or $\mathrm{n}^{\circ} 167$, Georgette Guéritey, épouse Marin : réquisitoire définitif du commissaire du Gouvernement près la cour de justice de la Côte-d'Or, le 31 janvier 1945. 
vue " après qu'elle a été tondue ${ }^{30}$. Quelques semaines plus tard, le commissaire du Gouvernement de la cour de justice de la Côte-d'Or explique que la sentence n'a pas été appliquée du fait de l'arrivée imminente des troupes alliées et du désir de l'inculpée qui réclamait " un conseil de guerre, siégeant dans des conditions normales ". Sans négliger cette affirmation, on peut néanmoins se demander si les résistants n'ont pas eu des doutes sur la culpabilité de Georgette Guéritey, et ce malgré ses aveux. Ont-ils préféré ne pas endosser la responsabilité de l'exécution d'une femme mère d'un jeune enfant - quand bien même le père est allemand - et préféré à leur sentence le travail d'un tribunal régulier? Le procès ne débouchant pas sur une exécution, les résistants semblent gênés et ne plus savoir que faire des captives. Un emprisonnement de longue durée, qui nécessite du personnel, des moyens financiers et un bâtiment spécialisé, leur semble impossible à gérer et ne peut finalement être pris en charge que par le nouveau pouvoir républicain. Ici, la transition entre la captivité et la détention s'effectue presque sans accroc. Les FFI remettent l'inculpée aux mains des autorités militaires de Dijon, qui l'enferment ensuite à la prison départementale. Remise en liberté un peu trop rapidement, profitant probablement du tumulte dû à la multiplication des arrestations dans les mois qui suivent la Libération, elle est de nouveau détenue à partir de janvier 1945 jusqu'à son procès le mois suivant. Jugée par une cour de justice, elle est une nouvelle fois condamnée à mort. Dans ce cas, le temps du jugement interrompt celui de l'emprisonnement : le procès est une étape, à la différence notable que, le verdict rendu, l'inculpée devient une prisonnière de longue durée, pour qui la liberté semble inaccessible.

Contrairement à la captivité, l'internement administratif et la détention ne servent pas uniquement à contenir des individus dangereux : ils permettent également de placer des suspects en sécurité. En septembre 1944, Wanda Zboinsky, qui fait l'objet d'un mandat d'arrêt du juge d'instruction près la cour de justice de Saint-Brieuc pour dénonciation et complicité d'assassinat d'un habitant de Loudéac, est arrêtée par la gendarmerie de Landivisiau. Prévenu, le frère de la victime, lui aussi Loudéacien, se propose d'aller la chercher avec son propre véhicule. Flanqué de deux agents de police auxiliaires, il se rend dans la commune finistérienne avec pour mission de conduire la prisonnière au juge d'instruction mais, sur la route le menant à Saint-Brieuc, il décide de passer par Loudéac ${ }^{31}$. Le 17 septembre au soir, Wanda Zboinsky est emprisonnée dans la chambre de sûreté de la brigade de gendarmerie locale. Le lendemain, le même conducteur signale que sa voiture est en panne, ce qui ne fait que retarder le départ pour Saint-Brieuc et laisse le temps à l'information de se diffuser : la délatrice est au cœur de la communauté. Très vite, " une foule qui peut être évaluée

30. Arch. dép. de la Côte-d'Or, 29 U 2/14, procès-verbal du jugement de l'inculpée par le conseil de guerre FFI, groupe " Les Cras », le 7 septembre 1944.

31. Prétextant un manque de carburant, argument difficilement recevable au vu du détour effectué pour se rendre à Loudéac. 
à environ 450 personnes s'est massée aux abords de la caserne pour voir cette femme et la conspuer ${ }^{32}$ ». Si un gendarme précise qu'il s'agit d'une " manifestation paisible d'une foule heureuse de voir cette femme arrêtée " et qu'aucun coup n'est porté à son encontre, l'adjudant commandant la brigade est tout de même contraint d'établir un service d'ordre pendant que l'inculpée monte en voiture sous les cris de la population qui réclame sa mort. Celle-ci est finalement prononcée par la cour de justice de SaintBrieuc le 12 juillet $1945^{33}$. Ici, le temps de détention provisoire a permis d'enquêter finement sur la collaboration de l'inculpée, de la condamner... et d'éviter une éventuelle exécution, qui aurait été injustifiée. En effet, l'inculpée n'était pas seule sur le banc des accusées. Mariette Masse, également condamnée à mort dans cette affaire, n'avait eu de cesse de faire porter la responsabilité de la délation sur les épaules de sa co-inculpée lors de l'instruction et de l'audience; quelques mois plus tard, elle reconnaît sa seule et unique culpabilité. Tandis que Zboinsky est libérée dès décembre 1946, Masse passe près de six ans derrière les barreaux, avant de bénéficier d'une libération conditionnelle. Comme on peut l'observer, le temps d'incarcération peut être mis à profit par les condamnées, qui se retrouvent seules face à elles-mêmes afin de réfléchir à leurs dires, et donc être un nouveau temps propice aux révélations ${ }^{34}$.

Devant les juges d'instruction des cours de justice, lors d'une procédure contradictoire, les inculpées ont la parole. Certaines reviennent sur leurs aveux en affirmant qu'ils ont été obtenus par la force. Sans exclure des débordements de la part des résistants, des policiers ou des gendarmes en charge des premiers interrogatoires, le caractère presque systématique avec lequel cet argumentaire est utilisé, de même que les contradictions des détenues, laissent penser à un système de défense formé "à chaud ". En tentant de mettre en avant les bavures dont elles auraient été victimes, elles espèrent disqualifier l'origine de leur enfermement et, par voie de conséquence, l'ensemble de l'enquête ainsi que le verdict qui viendra la clôturer ${ }^{35}$. Le cas d'Eugénie Gloux est caractéristique de ce point de vue. Arrêtée au début du mois de septembre 1944, puis internée dans le camp de Sarzeau, elle voit son dossier examiné par la commission régionale de vérification des décisions administratives le 4 décembre ${ }^{36}$. Renvoyée devant la justice, elle est placée en détention et finalement condamnée à la peine de mort par la cour de justice d'Ille-et-Vilaine, le 6 septembre 1945, en tant

32. Arch. dép. d'Ille-et-Vilaine, 214 W 26, dossier de la cour de justice des Côtes-du-Nord $n^{\circ} 167$, Wanda Zboinsky, épouse Adamzack : procès-verbal de la Gendarmerie nationale, déclaration du maréchal des logis chef Victor Caulet, le 18 septembre 1944.

33. Arch. dép. d'Ille-et-Vilaine, 214 W 26, idem.

34. Arch. dép. d'Ille-et-Vilaine, 214 W 26, dossier de la cour de justice des Côtes-du-Nord $\mathrm{n}^{\mathrm{o}} 167$, Mariette Masse.

35. Voir sur ce point l'ouvrage dirigé par Chauvaud, Frédéric, Pretou, Pierre (dir.), L'arrestation. Interpellations, prises de corps et captures depuis le Moyen Âge, Rennes, PUR, 2016.

36. À partir du dernier trimestre de l'année 1944, on adjoint une commission régionale de vérification des internements à la commission départementale. 
qu'agent de renseignement au service de l'occupant. Un gendarme certifie qu'elle lui aurait avoué son appartenance à la Gestapo lors d'un interrogatoire. Faux, répond l'inculpée, ajoutant avoir été rouée de coups. À bout de forces, elle aurait dit au gendarme qui prenait sa déclaration d'écrire ce qu'il souhaitait. Sentant sans doute qu'elle ne convainc pas son interlocuteur, elle ajoute : "Je suis même restée deux jours et deux nuits sans nourriture et sans boisson jusqu'au moment où les gendarmes m'ont trouvée inanimée sur le ciment de ma cellule ${ }^{37}$. " La détention est clairement assimilée à une torture. Selon l'inculpée, ses aveux seraient sans valeur car, même s'ils n'ont pas été obtenus par la violence, la faiblesse de son état physique laisse penser qu'elle n'était pas en capacité de savoir ce qu'elle disait et qu'il lui est désormais impossible de s'en souvenir.

\section{La prison : un enjeu de l'épuration}

Durant les premiers mois de la Libération, même une fois le jugement passé, l'emprisonnement demeure un temps marqué du sceau de l'incertitude en raison des critiques dont la justice est la cible. Les résistants et les populations reprochent à l'épuration judiciaire sa trop grande magnanimité et n'hésitent pas à forcer les portes des prisons pour s'emparer de prisonnières dont ils estiment l'incarcération injustifiée. Pour les anciens collaborateurs, une seule issue s'impose : la mort. C'est ainsi que dans le courant du mois de novembre 1944, suite à une attaque meurtrière, les FFI casernés dans le château de La Simone, en Vaucluse, une vingtaine de personnes suspectées d'être à l'origine de l'attentat sont brutalisées et emprisonnées à Pertuis. L'émotion et les tensions étant à leur paroxysme dans la région, un groupe du régiment FFI de Digne attaque la prison de la ville et s'empare de Marie-Louise Ratier ainsi que d'un autre détenu, tous deux condamnés à la peine de mort par la cour de justice des Basses-Alpes. Tandis qu'un pourvoi en cassation a été formé et que la demande de grâce présidentielle suit son chemin, les résistants anticipent une éventuelle décision favorable aux condamnés : sortis de prison, les deux individus sont passés par les armes. De toute évidence, lorsque les murs des prisons sont encore friables et que celles-ci deviennent le théâtre de violences, c'est que la légitimité de droit peine à se rétablir dans certaines régions de France, principalement situées dans l'ancienne zone libre ${ }^{38}$. Une seconde vague de violences, qui se manifeste également par des attaques de prisons, est très nettement perceptible lors du rapatriement des déportés au printemps et à l'été 1945, preuve s'il en est que ces dernières demeurent au cœur des tensions entre épuration judiciaire et extrajudiciaire. Ces soubresauts évités,

37. Arch. dép. d'Ille-et-Vilaine, $213 \mathrm{~W} 42$, dossier de la cour de justice d'Ille-et-Vilaine $\mathrm{n}^{\circ}$ 145, Eugénie Gloux, épouse Pottier.

38. Arch. dép. des Alpes-de-Haute-Provence, $9 \mathrm{~W} 1$ : lettre du procureur de la République délégué pour le procureur général, le 7 février 1945. Voir également à ce propos, l'étude très fouillée de MENCHÉRINI, Robert, Midi rouge, ombres et lumières, tome 4 : La Libération et les années tricolores (1944-1947), Paris, Syllepse, p. 263 et suivantes. 
débute une incarcération qui s'inscrit dans la lignée du temps de détention : la parole des femmes emprisonnées continue d'être écoutée. En compagnie de leurs avocats, elles peuvent s'appuyer sur l'arsenal juridique existant pour contester leur jugement et leur emprisonnement.

À cet effet, rares mais néanmoins notables sont celles qui tentent de faire jouer les accords internationaux. Inculpée d'espionnage et de commerce avec la Wehrmacht, l'Allemande Elisabeth Friess mobilise ainsi les termes de la convention de La Haye pour obtenir le droit au traitement des prisonniers de guerre. D'après elle, son affaire échappe à la compétence de la cour de justice car elle s'inscrit " à la suite de l'armée d'occupation ". Après avoir étudié le droit international public en la matière, le commissaire du Gouvernement certifie le contraire. Effectivement, des non-combattants (comme les vivandiers, les portefaix ou les correspondants de journaux) ont le droit au statut de prisonniers de guerre car ils entrent dans la composition de l'armée, la suivent dans tous ses déplacements et en ont obtenu la légitimation; mais davantage que des prisonniers de guerre, Elisabeth Friess et ses co-inculpés relèvent de la catégorie des " personnes capturées ", telle que définie par le droit actuel des conflits, car ils ont commercé librement avec la Wehrmacht et ont entretenu des relations très cordiales avec les chefs de la Gestapo ${ }^{39}$. Certes, ils étaient chargés du ravitaillement des forces allemandes mais ils vivaient de façon autonome, tel un organisme privé, sans aucun lien de subordination avec les services allemands, qui leur accordaient simplement une "bienveillante protection ". De plus, ils ont constamment demeuré à Lille de 1941 à 1944 en étant propriétaires de leur matériel et de leurs moyens de transport, en rétribuant leur personnel et en effectuant un commerce qui dépassait les frontières de la France occupée. Après avoir été condamnée à mort par la cour de justice du Nord en juillet 1945, Friess voit son jugement cassé en avril 1947, en raison non pas des accords de La Haye mais de sa nationalité. La chambre criminelle de la Cour de Cassation indique que lorsqu'un individu accusé d'espionnage appartient par sa nationalité au pays avec lequel il a entretenu des intelligences avec l'ennemi, son affaire relève des tribunaux militaires. Difficile effectivement de reprocher l'esprit de collaboration à cette femme qui, devant un tribunal militaire, sera jugée pour d'autres types d'incrimination ${ }^{40}$. Ce dernier, contrairement à une cour de

39. Sur cette différence entre " prisonniers de guerre " et " personnes capturées ", nous renvoyons le lecteur à GENET-RoufFIAC, Nathalie (dir.), Des prisonniers de guerre aux personnes capturées, Paris, Service historique de la Défense, 2010.

40. Centre des Archives contemporaines de Fontainebleau, 19850499/163, dossier 23658 S 45, Élisabeth Friess, épouse Berndt : arrêt de la Cour de cassation, chambre criminelle, le 17 avril 1947 et lettre du commissaire du Gouvernement près la cour de justice du Nord pour le garde des Sceaux, le 6 août 1945. Cet arrêt fera jurisprudence : voir "Les atteintes à la sûreté extérieure de l'État commises par des ressortissants ennemis ", Revue de science criminelle et de droit pénal comparé, $\mathrm{n}^{\circ}$ 4, octobre-décembre 1947, p. 44, ainsi que Garçon, Émile (dir.), Code pénal annoté, Paris, Sirey, 1952, tome 1, p. 249-250, qui note : "Sans doute les accords de La Haye de 1907 ont-ils édicté des règles nettes en ce qui concerne les personnes appartenant aux armées ennemies, mais il resterait peut-être 
justice qui n'est compétente qu'en matière de collaboration, peut notamment juger les ressortissants allemands pour crimes de guerre.

Pour ne pas connaître l'incarcération, des femmes choisissent la fuite afin de se cacher en France ou de s'exiler. Quelques-unes poursuivent ou refont leur vie quand d'autres vivent cette expérience comme une captivité à ciel ouvert. Loin des leurs, confrontées à la misère et à l'intolérance, avec de faibles perspectives d'avenir, l'exil est par exemple mal vécu par les Bretonnes ayant fait le choix de s'installer en Irlande à la sortie de la guerre $^{41}$. C'est en partie parce que l'exil rime avec contraintes et difficultés que des réprouvées condamnées à mort par contumace frappent à la porte de la justice française au début des années 1950. Rassurées par la promulgation des trois principales lois d'amnistie de la collaboration (en 1947, 1951 et 1953) qui rendent impossible la condamnation de beaucoup des faits qui leur sont reprochés, elles se rendent. Les détentions préventives sont alors considérées comme inutiles par les juges d'instruction qui répondent favorablement aux demandes des avocats afin que leurs clientes demeurent en liberté provisoire. Ils ne jugent sans doute pas l'emprisonnement indispensable à la manifestation de la vérité ou ne se font guère d'illusions sur l'issue de ces ultimes procès de l'épuration, qui revêtent davantage un caractère désépuratoire.

À l'inverse, cette liberté provisoire n'est qu'exceptionnellement accordée aux femmes (r)attrapées par l'épuration et jugées dans l'immédiat après-Libération. Condamnées à la peine capitale mais graciées, elles ont dû s'habituer à la vie quotidienne en prison.

\section{Le quotidien des femmes incarcérées}

Une fois condamnées à mort, les inculpées rejoignent leur cellule ${ }^{42}$. Leur incarcération se divise alors en deux périodes d'inégale importance. La première débute lorsque le verdict de la juridiction civile ou militaire tombe, et se termine une fois la réponse au recours en grâce obtenue. En

à enfermer dans des limites plus rationnelles l'inculpation d'intelligence avec l'ennemi quand le crime est reproché à des civils étrangers. "

41. Nous renvoyons à ce sujet aux travaux de Sébastien Carney, et notamment à sa communication "Les épurés du mouvement breton en Irlande : l'interceltisme à l'épreuve de la réalité ", présentée au colloque organisé les 11 et 12 juin 2015 à l'université Rennes 2, sous la direction de Marc Bergère, intitulé Pour une histoire connectée et transnationale des épurations en Europe au sortir de la Seconde Guerre mondiale.

42. Nous possédons quelques descriptions de la vie des hommes condamnés à mort dans la prison de Fresnes. Voir notamment le témoignage du détenu infirmier, BocognAGO, Jean, Quartier des fauves, Paris, éditions du Fuseau, 1953, ou celui du condamné à mort BÉRAUD, Henri, Quinze jours avec la mort. La chasse au lampiste, Paris, Plon, 1951. Voir également les travaux des historiens VIMONT, Jean-Claude, "Les fers des condamnés à mort... ", art. cit. et PICARD, Nicolas, "Corps enchaînés, surveillés et découpés. Les contraintes matérielles des condamnés à mort en France au Xx ${ }^{\mathrm{e}}$ siècle ", dans PORRET, Michel, Fontana, Vincent, Maugue, Ludovic (dir.), Bois, fers et papiers de justice..., op. cit., p. 282-298. 
général, leur peine est commuée en travaux forcés à perpétuité. Débute un second temps, celui de l'incarcération de longue durée. Effectuée dans des maisons centrales réservées aux femmes, comme celle de Rennes, elle est marquée par des rythmes spécifiques, entre attente et tensions, entre souffrances et espoir, mais également par des punitions qui témoignent des craintes dont les anciennes collaboratrices sont l'objet de la part de l'administration pénitentiaire.

\section{Purger sa peine : entre linéarité du temps carcéral et rupture de la vie privée}

"Rien ne se dessine, ni la mort ni la grâce. " Le 10 mars 1949, depuis le quartier de haute-surveillance de la prison de Fresnes, Mathilde Carré écrit dans un carnet, qui servira ensuite de base à la rédaction de ses mémoires, d'intéressants renseignements sur ce qui fait l'essentiel d'une vie de prisonnière condamnée à mort : l'attente ${ }^{43}$. Pour débuter, l'attente de la grâce présidentielle, puis dans le cas d'une réponse positive à cette première sollicitation, celle des remises gracieuses et des nouvelles des proches. Jugée deux mois plus tôt, elle est, comme toutes les femmes incarcérées à cet instant, dans l'expectative. Après le rejet du pourvoi en cassation et pendant que leurs avocats tentent de les défendre auprès du chef de l'État, la cellule fait figure de sas pour les condamnées à mort, entre privation de liberté et exécution. Sas dans lequel le vécu et la perception diffèrent selon les prisonnières, et parfois chez une même prisonnière au gré des événements et des moments. Plusieurs semblent abattues quand d'autres crient encore leur innocence ou, du moins, contestent une peine leur paraissant exagérée. L'allongement du temps d'attente n'augure rien de bon et fait augmenter l'angoisse. Surtout que les exemples d'exécution ne sont jamais loin. Mathilde Carré note par exemple : "Ce matin, à l'aube, le sinistre et bruyant cortège qui vient chercher un condamné à mort, Jean Bassompierre ${ }^{44}$. " La nécessité ressentie par l'espionne de coucher sur le papier cet événement prouve son importance - sans doute due à la personnalité exécutée, même si l'auteur prétend ne pas la connaître - et la formulation utilisée son intégration. Le bruit évoqué ne semble pas la surprendre outre mesure, comme si ces extractions vers la mort, après plusieurs années d'enfermement, lui étaient désormais familières et banales; elles troublent à peine sa vie réglée de prisonnière.

43. CARRÉ, Lily, On m'appelait la chatte..., op. cit., p. 260, à la date du 10 mars 1949. Sur la prison de Fresnes à la Libération, se reporter à Bénédicte VERGEZ-ChAignon, Vichy en prison..., op. cit.

44. CARré, Lily, On m'appelait la chatte..., op. cit., p. 239, à la date du 20 avril 1948. Jean Bassompierre est à l'origine du Service d'ordre légionnaire et l'un des piliers du mouvement avec son ami Joseph Darnand. À la dissolution du Service d'ordre légionnaire, il rejoint la LVF puis, en février 1944, il réorganise la Milice française dans la zone nord. En juillet 1944, il réduit la mutinerie de la prison de la Santé. Il fuit en Allemagne en août 1944 et y intègre la Division Charlemagne. Condamné à mort par la cour de justice de la Seine en janvier 1948, il est fusillé quatre mois plus tard. 
Afin de rompre le quotidien, de garder contact avec le monde extérieur et de se rattacher à ce qui faisait leur identité de filles, de mères, d'épouses ou encore de travailleuses, les prisonnières échangent de nombreux courriers. Ceux-ci démontrent que leur situation connaît d'importants bouleversements. En 1950, Eugénie Gloux, en prison à Rennes depuis cinq ans, écrit au commissaire du Gouvernement afin de solliciter une mesure gracieuse. Elle met en avant sa volonté de se racheter en disant qu'une fois libérée, elle sera une mère exemplaire. C'est d'ailleurs pour ses enfants, qui l'attendent, qu'elle écrit et demande pitié. Surtout, elle sent que son " mari ne poura pas matendre encore des année $[s i c]^{45}$ ". Effectivement, quelques mois plus tard, celui-ci engage une action en divorce et sollicite auprès du Procureur général une copie de la décision prononçant la condamnation à mort de son épouse afin de justifier auprès du tribunal civil le bien-fondé de sa demande ${ }^{46}$. Que la volonté de séparation tire son origine de la collaboration ou que l'épuration purge des conflits plus anciens, les liens du mariage ne résistent pas toujours à l'épreuve de l'emprisonnement.

Pour quelques individus, le divorce ne permet pas seulement de couper les liens avec le conjoint; il est également une occasion de se dédouaner. Albert Ceugniez dit avoir introduit, " comme cela va de soi ", une instance de divorce après avoir appris l'incarcération de sa femme, la collaboratrice rennaise Adrienne Tullou. Lui-même prisonnier de guerre à partir de 1940, il était devenu travailleur volontaire en Allemagne; fervent admirateur du régime national-socialiste, il avait écrit à son épouse pour l'inviter à venir le rejoindre en Allemagne. Adrienne Tullou profite quant à elle de cette demande de divorce pour intégrer les lettres de son mari à son système de défense : elle insiste sur son refus systématique de répondre favorablement aux sollicitations réitérées de son mari qui, contrairement à elle, était un collaborateur acharné ${ }^{47}$. Entre vie privée et enjeux politiques, cette séparation permet de circonscrire l'engagement de chacun et constitue un nouveau système de défense pour la prisonnière, lui permettant de rejouer son procès et de contester la sanction qui lui a été infligée.

Outre les échanges épistolaires, des permis de visite peuvent être attribués par le préfet. Ceux de Lucienne Besnier, condamnée à mort par la cour de justice du Maine-et-Loire, nous apprennent qu'elle a reçu à la maison d'arrêt d'Angers, de décembre 1945 à novembre 1948, 66 visites de quatre membres de sa famille habitant à proximité, soit pratiquement deux visites

45. Arch. dép. d'Ille-et-Vilaine, $213 \mathrm{~W} 42$, dossier de la cour de justice d'Ille-et-Vilaine $\mathrm{n}^{\circ}$ 145, Eugénie Gloux, épouse Pottier : lettre de l'inculpée pour le commissaire du Gouvernement, le 29 janvier 1950.

46. Arch. dép. d'Ille-et-Vilaine, $213 \mathrm{~W} 42$, lettre de $\mathrm{M}^{\mathrm{e}}$ Huchet, avocat de M. Pottier, pour le Procureur général, le 25 janvier 1952.

47. Arch. dép. d'Ille-et-Vilaine, $213 \mathrm{~W} 22$, dossier de la cour de justice d'Ille-et-Vilaine $\mathrm{n}^{\circ} 6$, Adrienne Tullou, épouse Ceugniez : procès-verbal de la police nationale, audition de l'inculpée, à Rennes, le 29 septembre 1944; et 213 W 43, dossier de la cour de justice d'Ille-et-Vilaine $\mathrm{n}^{\circ} 147$, Adrienne Tullou, épouse Ceugniez : lettre d'Albert Ceugniez pour le commissaire du Gouvernement près la cour de justice d'Ille-et-Vilaine, le 7 mai 1946. 
par mois ${ }^{48}$. Cette fréquence demande toutefois à être considérablement nuancée en fonction de la distance qui sépare le lieu d'enfermement de la condamnée - il change régulièrement - du lieu de résidence de la famille et des moyens dont dispose cette dernière afin de se déplacer. Rares sont les proches déménageant pour être au plus près de la prisonnière, comme la mère de Georgette Guéritey qui quitte la Côte-d'Or pour s'installer à Rennes ${ }^{49}$. Parfois, les visites et les lettres ne suffisent pas à lutter contre le sentiment d'abandon. De là sans doute la punition infligée à la femme Hollande; cette prisonnière a voulu prolonger la discussion engagée avec sa famille venue lui rendre visite depuis le Nord-Pas-de-Calais, en dépassant la durée réglementaire du parloir. Le temps de discussion autorisé paraît bien court aux membres d'une famille qui ne se sont pas vus depuis plusieurs mois. Dans le même esprit, le bavardage est de loin le fait le plus sanctionné par les surveillantes de la maison centrale de Rennes, preuve s'il en est de l'impérieux besoin ressenti par les prisonnières d'échanger ${ }^{50}$.

\section{Des femmes entre ordre carcéral et ordre moral}

Quand on observe les avis formulés par le directeur de cette prison sur le comportement des femmes, afin que les autorités judiciaires puissent se positionner par rapport aux demandes de mesures gracieuses, une image se dégage très nettement : celle d'incarcérées modèles, qui expient leurs fautes et travaillent consciencieusement. La confrontation de ces avis avec le «bulletin de statistique morale " présent dans chaque dossier de prisonnière confirme généralement cette bonne volonté. Pour autant, la réalité est parfois plus complexe.

Au-delà de l'attitude des prisonnières, ces bulletins nous informent sur les inquiétudes de l'administration pénitentiaire quant à leurs pratiques sexuelles. À mots plus ou moins couverts, l'explication de sanctions infligées par les surveillantes laisse transparaître plusieurs cas d'homosexualité, probable pratique d'attente pour des femmes " à qui manquent tellement les hommes ${ }^{51}$ ». En décembre 1948, l'une d'entre elles est réprimandée pour " avoir rapproché son lit de sa compagne et mis ses couvertures en commun $^{52}$ ». Une deuxième est mise au pain sec pendant deux jours car

48. Arch. dép. d'Ille-et-Vilaine, 278 W 35, dossier 659, numéro d'écrou 685 : permis de visite permanents.

49. Centre des Archives contemporaines de Fontainebleau, 20070634/20, dossier $1602 \mathrm{~S}$ 55, Georgette Guéritey, épouse Marin : lettre de l'inculpée au Président de la République, le 28 août 1956.

50. Arch. dép. d'Ille-et-Vilaine, 278 W 246, dossier 4282, numéro d'écrou 8 : bulletin de statistique morale.

51. CARré, Lily, On m’appelait la chatte..., op. cit., p. 224, à la date du 14 juillet 1947, ainsi que le stimulant article de ThÉOFILAKIS, Fabien, "La sexualité du prisonnier de guerre. Allemands et Français en captivité (1914-1918, 1940-1948) ", Vingtième siècle. Revue d'histoire, $\mathrm{n}^{\circ}$ 99, 2008/3, p. 203-219.

52. Arch. dép. d'Ille-et-Vilaine, 278 W 124, dossier 2247, numéro d'écrou 1866 : bulletin de statistique morale. 
elle bavarde après l'extinction des feux avec une autre prisonnière, qui l'a embrassée. Enfin, deux autres femmes sont séparées car elles partagent ensemble des "mœurs spéciales ${ }^{53}$ ". Ces remarques rejoignent celles de " la chatte " lorsqu'elle s'offusque en voyant des "Jules " et leurs " Julies " qui " se faufilent d'un lit à l'autre dès que tout semble endormi, se chamaillent jalousement, gémissent ", avant de préciser que ces relations ne concernent pas exclusivement les prisonnières puisque l'une d'entre elles est " en ménage avec une surveillante ${ }^{54}$ ". Comme l'ont démontré les historiens travaillant sur le stéréotype du collaborateur à la Libération, la demande d'épuration associe souvent la trahison à une forme de dégénérescence morale et/ou sociale : aux yeux des communautés, la trahison ne peut venir que d'êtres hors-normes, d'individus " dénaturés ${ }^{55}$ ". Davantage que d'autres prisonnières, les collaboratrices sont donc au cour des préoccupations liées à la sexualité. Contre-modèles des bonnes Françaises, épouses fidèles et mères dévouées, leur homosexualité prouve qu'elles ne s'amendent pas et qu'elles ne sont pas prêtes à retrouver la place qui leur est dévolue dans la société. Les collaboratrices continuent de représenter cette France défaite et couchée symbolisée à la Libération par la collaboration sexuelle. Dès lors, dans ce corps social en plein redressement que forme la nation française, celui des prisonnières est à redresser tant moralement que symboliquement.

D’une manière générale, l'emprisonnement est marqué par la perte d'identité et l'absence d'intimité. Mise à nu puis revêtue d'une robe en " grosse bure marron ", d'une " couche-culotte boutonnée entre les jambes " et enchaînée par les pieds, "la chatte " se plaint de ces surveillantes qui, à Fresnes, "lui ont retiré tout ce qui [lui] appartenait ${ }^{56}$ ". Cette scène se renouvelle quelque temps plus tard à son entrée dans la prison rennaise, les fers et les menottes en moins depuis que la grâce a été obtenue ${ }^{57}$. Rien d'étonnant donc à ce que les femmes incarcérées effectuent des demandes afin de récupérer leurs vêtements, conservés à la lingerie de l'établissement pénitentiaire. Faire pénétrer quelques effets personnels dans ce monde uni-

53. Arch. dép. d'Ille-et-Vilaine, 278 W 122, dossier 2208, numéro d'écrou 11419 : bulletin de statistique morale et 278 W 132, dossier 2406, numéro d'écrou 1868, bulletin de statistique morale.

54. CARRÉ, Lily, On m'appelait la chatte..., op. cit., p. 276, septembre 1950.

55. Sur le stéréotype du collaborateur, voir BERGĖRE, Marc, Une société en épuration..., op. cit., p. 334-344 et CAPDEVILA, Luc, Les Bretons au lendemain de l'Occupation..., op. cit., p. $356-372$.

56. CARRÉ, Lily, On m'appelait la chatte..., op. cit., p. 253, à la date du 12 janvier 1949 .

57. Avant une éventuelle grâce, les condamnées à mort sont placées dans un quartier de haute surveillance. Surveillées de manière permanente afin d'éviter toute tentative d'évasion ou de suicide, elles sont enchaînées et menottées (PICARD, Nicolas, " Des fauves en cage : reportages et témoignages sur les condamnés à mort dans la presse française (années 1920-1950) ", Revue Circé. Histoires, cultures et sociétés [en ligne], n ${ }^{\circ} 5$, juin 2014, [http://www.revue-circe.uvsq.fr/des-fauves-en-cage-reportages-et-temoignages-surles-condamnes-a-mort-dans-la-presse-francaise-annees-1920-1950/], consulté le 23 décembre 2016). 
formisé qu'est la prison permet de se démarquer des autres prisonnières et de se réapproprier une identité passée. Enfin, cela permet aux prisonnières de contrer la négation de leur féminité imposée par le port de tenues uniformes. De ce point de vue, le manque de respect envers les surveillantes suite à leurs remarques pour infraction au règlement peut également être considéré comme un espace, d'indépendance et de liberté.

Dans la perspective de ce redressement-amendement, la religion est une pratique particulièrement encouragée dans les prisons de femmes. Réconfort dans un monde empreint d'agressivité et face à l'incertitude d'un futur non maîtrisé, la religion est régulièrement évoquée dans les mémoires de Mathilde Carré. On peut y lire que « l'influence de la Bretagne bigote pénètre jusque dans nos murs ". Après avoir longtemps manifesté sa défiance à l'égard des convictions chrétiennes, l'espionne décide de se faire baptiser en juin 1953, plus de quatre ans après sa condamnation à mort. Sans doute l'influence des religieuses qui surveillent les femmes de la Roquette, où elle a été transférée à la fin de l'année 1952, le mépris dont elle a été la cible à l'évocation de son athéisme, comme le fait d'avoir échappé à l'exécution, ne sont pas étrangers à cette foi naissante. Cependant, si à "Rennes-la-pieuse " comme dans d'autres prisons, les cérémonies religieuses encadrent la vie des prisonnières, il ne faudrait pas conclure de façon hâtive que l'ensemble des prisonnières se réfugient dans les bras de l'Église ${ }^{58}$. La réalité est plus complexe. Tandis que l'emprisonnement ne modifie en rien la force de la foi ou de l'athéisme de beaucoup de femmes, il démontre les hésitations ou la superficialité des croyances de plusieurs d'entre elles. En 1947, Marcelle Dulevron reconnaît par exemple avoir demandé à ne plus assister aux offices parce qu'elle était déprimée et surtout car elle y avait subi une mauvaise influence. Mais là n'est pas l'essentiel : en croisant cette lettre avec d'autres, on apprend qu'elle a cherché une tutrice parmi les religieuses qui ont toutes décliné son offre. Vexée, elle s'est détournée des catholiques pour s'adresser au pasteur de la prison ${ }^{59}$. Une autre prisonnière demande à être dispensée d'offices car elle n'y trouve nullement " l'aide et le secours spirituel » dont elle a besoin; au contraire, elle dit se sentir un peu plus découragée chaque fois qu'elle entend parler l'aumônier. Comme Dulevron, elle demande alors à assister aux offices protestants ${ }^{60}$. Dans un autre état d'esprit car elle ne fait pas succéder une confession à une autre, Léontine Christollet ne souhaite plus se rendre aux célébrations car, bien que catholique, elle s'est toujours abstenue de toute pratique. Afin d'être bien certaine d'obtenir gain de cause auprès de la direction, elle met en avant son état de santé qui ne

58. CARRÉ, Lily, On m'appelait la chatte..., op. cit., p. 278 et 280, septembre 1950 et 1951 , pour les deux dernières citations.

59. Arch. dép. d'Ille-et-Vilaine, 278 W 157, dossier 2832, numéro d'écrou 1091 : lettres de la prisonnière au directeur de la maison centrale, toutes datées de 1947.

60. Arch. dép. d'Ille-et-Vilaine, 278 W 251, dossier 4360, numéro d'écrou 842 : lettres de la prisonnière au directeur de la maison centrale, les 2 et 24 mars 1947. 
lui permet pas de rester debout très longtemps à la chapelle ${ }^{61}$. De cette façon, elle affirme qu'elle peut très bien se passer de la religion et de ses sociabilités, même lorsqu'elle est enfermée. En fait, c'est surtout lorsque la perspective d'une sortie de prison est évoquée que l'aide ecclésiastique est primordiale. Des œuvres, telles que la maison Marie-Jean-Joseph à Villejust, dans la banlieue sud de Paris, fournissent en effet les précieux certificats d'hébergement et de travail sans lesquels les condamnées à mort ne peuvent bénéficier d'une libération conditionnelle. Entre volonté personnelle et manque de réseaux extérieurs, ces femmes bénéficient donc de l'aide de l'Église, ce qui rassure l'administration pénitentiaire. Avec la certitude que ses anciennes pensionnaires seront bien entourées, celle-ci transmet sans hésiter le témoin de l'amendement aux œuvres religieuses. Le passage d'une vie marquée par la contrainte à un univers de liberté relativement surveillée n'est jamais une rupture nette mais plutôt une transition, pour des femmes qui ont des comptes à rendre durant tout le temps de leur libération conditionnelle. Quelques-unes donnent des nouvelles depuis leur centre d'accueil, sans regrets bien entendu pour la vie carcérale, mais avec un mot de reconnaissance ou, a contrario, quelques reproches à adresser au personnel de la prison.

\section{Un milieu carcéral inégalitaire}

La bonne volonté dont font preuve beaucoup de femmes ne saurait masquer les tensions à l'intérieur de la prison. Les affaires ne se terminent pas une fois l'arrestation effectuée et encore moins le procès achevé. En avril 1946, Charlotte Weyl se plaint de Denise Dayet, une condamnée à mort enfermée à Rennes entre 1951 et 1955, qui se vanterait d'avoir connaissance de toute l'affaire du service de renseignement allemand de Dijon et de " faire tout son possible pour [la] faire entrer " dans cette affaire ${ }^{62}$. Les jeux de pouvoir propres à la vie carcérale s'appuient sur le passé collaborateur des inculpées qui, en retour, avive les conflits. Du coup, les insultes et les disputes ne sont pas rares. Le 22 septembre 1948, Yvonne Caillard est condamnée à deux jours de pain sec après une altercation avec une autre prisonnière de la centrale bretonne; un mois plus tard, elle subit la même peine et est déclassée pour avoir calomnié une autre condamnée à mort. Cependant, les frictions n'éclatent que rarement entre celles qui se nomment " les politiques". Outre les distinctions qui s'opèrent en fonction de la nature de la collaboration et des convictions de chaque femme, les " politiques " ont conscience de former une communauté à part durant leur emprisonnement. À mesure de l'élargissement des épurées, la population carcérale redevient majoritairement composée des criminelles de

61. Arch. dép. d'Ille-et-Vilaine, 278 W 94, dossier 1743, numéro d'écrou 1082 : lettre de la prisonnière au directeur de la maison centrale, le 2 février 1947.

62. Arch. dép. de la Côte-d'Or, 29 U 76, dossier Denise Dayet : procès-verbal d'audition de Charlotte Weyl et confrontation avec l'inculpée, devant le juge d'instruction Bouchard, le 23 avril 1946. 
" droit commun et de mineures " précise Mathilde Carré, avant d'ajouter qu'elles " reprennent possession de cette maison, qui était la leur ${ }^{63}$ ». Si les " traces " de la collaboration semblent disparaître plus rapidement derrière les murs des prisons que dans les mémoires de la population française, une nouvelle fois, cette manière qu'ont les anciennes collaboratrices de vivre leur emprisonnement ne correspond pas à ce que doit être leur vie après la prison. Malgré l'instruction en date du 12 avril 1945 précisant que les condamnées pour faits de collaboration " doivent être soumis[es] au même régime que celui qui est imposé par les règlements en vigueur aux différentes catégories de condamnés et qui diffère suivant la nature de la peine prononcée ", elles demeurent à part, soit parce qu'elles sont exclues par les autres prisonnières, soit parce qu'elles-mêmes ne souhaitent pas se mêler au reste de la population carcérale ${ }^{64}$. Dans le même esprit, dans un recours en grâce effectué en 1948, Marie-Louise Degoul proteste au nom de toutes les innocentes condamnées pour des faits de collaboration car elles subissent le même régime que celui des "droits communs " en centrale, c'est-à-dire dans des " établissements qui viennent - Cayenne supprimé immédiatement après l'île de Ré65 ". Bien entendu, si l'argument du grand nombre d'innocentes qui purgent injustement une peine de prison ne peut être retenu par les autorités examinant cette demande, le contraste entre les grandes criminelles de droit commun et celles qui, au grand maximum, se présentent comme des petites mains de la collaboration, peut retenir leur attention. Inacceptable en 1944, cet argument devient audible quatre années après la Libération.

Beaucoup de prisonnières supportent très difficilement leur enfermement et oscillent entre lassitude et perte de contrôle. Anciennes et, pour une partie d'entre elles - dont la maison centrale de Rennes - gravement endommagées durant les bombardements ou les combats du printemps et de l'été 1944, les prisons françaises sont en piteux état à la Libération. L'insalubrité et le froid qui y règnent, ajoutés aux mauvaises conditions d'hygiène et au manque de nourriture, rendent la vie quotidienne des femmes incarcérées particulièrement difficile ${ }^{66}$. Si elles peuvent " cantiner » en travaillant à l'intérieur de la prison, l'essentiel de l'argent et des vivres supplémentaires dont elles disposent, à l'image des prisonniers de guerre français en Allemagne durant le premier ou le second conflit

63. CARrÉ, Lily, On m'appelait la chatte..., op. cit., p. 239, à la date du 20 avril 1948. Mathilde Carré évoque ici la prison de Fresnes.

64. GARÇON, Émile (dir.), Code pénal annoté, op. cit., p. 266. Toutefois, ce dernier précise ensuite, à la page 387 , que "l'administration pénitentiaire a toujours estimé nécessaire de séparer les condamnés de cours de justice des détenus de droit commun " et que des instructions ont même été données dans ce sens dès le mois d'avril 1945.

65. Centre des Archives contemporaines Fontainebleau, 20070194/193, dossier 14283 S 50, Marie-Louise Degoul, épouse Montagut : recours en grâce de l'inculpée pour le président du Conseil, le 19 septembre 1948.

66. FAUGERON, Claude, "De la Libération à la guerre d'Algérie : l'espoir d'une réforme pénitentiaire ", dans PetrT, Jacques Guy (dir.), Histoire des galères, bagnes et prisons XIII XXe siècles, Toulouse, Privat, 1991, p. 289. 
mondial, vient de l'extérieur ${ }^{67}$. La richesse de cet apport permet de changer leur train de vie et engendre des distinctions au sein de la population carcérale. Incontestablement, l'inégalité des ressources familiales et/ou conjugales se fait ressentir à l'intérieur des établissements pénitentiaires. Des prisonnières, comme Marcelle Hellenthal, se plaignent de leur état de santé déclinant, qui s'expliquerait essentiellement par l'absence de colis. En octobre 1948, elle dit souffrir " un martyr insupportable " à la prison de Rennes car elle est privée de tout et se trouve sans argent et sans colis " après une vie d'économies et de travail ". Elle précise que "d'une jolie fille, il ne reste plus qu'un cadavre ${ }^{68}$ ". Mathilde Carré, quant à elle, apprécie d'être bien assistée par ses parents : sa mère lui prépare des colis que son père vient lui apporter toutes les semaines, accompagnés d'un chèque déposé au greffe à la fin de chaque mois ${ }^{69}$. En revanche, elle signale que l'état de santé d'une prisonnière nommée Charlotte est très préoccupant car elle a constamment faim; ses parents sont pauvres et " ils se privent pour lui apporter un peu de pain et de pâté au parloir, chaque vendredi ${ }^{70}$ ". La nourriture est donc une préoccupation majeure dans la vie des prisonnières. En quantité suffisante, elle permet de maintenir un état physique satisfaisant et de garder le moral; elle rend donc la vie en maison centrale plus supportable et permet de se projeter sans craintes à moyen ou long terme. Disposer de davantage de nourriture contribue également à renforcer son influence sur les prisonnières moins bien loties de ce point de vue et de se débarrasser des tâches ingrates, ce qui n'est jamais superflu derrière les barreaux. On devine par exemple qu'une dénommée Marie-Jeanne lave les culottes, les chemisiers et les mouchoirs de "la chatte " après que celle-ci lui a fait profiter d'une partie de ses colis ${ }^{71}$. En conséquence, la privation de ces précieux apports est une sanction privilégiée par l'administration pénitentiaire.

67. La " cantine " désigne la possibilité pour un prisonnier d'acheter des produits de la vie courante tels que de la nourriture, des cigarettes, des timbres et des enveloppes, des produits d'hygiène, des journaux, etc. Les produits de cantine doivent être distingués des services fournis gratuitement par l'administration pénitentiaire. Par exemple, si le prisonnier peut cantiner de la nourriture, celle-ci vient en supplément des repas qui lui sont quotidiennement fournis par la prison. Pour une comparaison avec les prisonniers de guerre français durant la Seconde Guerre mondiale, se reporter notamment à DuRAND, Yves, La captivité. Histoire des prisonniers de guerre français 1939-1945, Paris, FNCPG, 1980 et Les prisonniers de guerre dans les Stalags, les Oflags et les Kommandos, Paris, Hachette, 1994; FISHMAN, Sarah, Femmes de prisonniers de guerre, 1940-1945, Paris, L'Harmattan, 1996 [1991]. Pour un exemple de prisonniers de guerre bretons en Allemagne durant la Grande Guerre, voir LAGADEC, Yann, "Deux expériences de la captivité de guerre : Paul Cocho et Élie Préauchat, territoriaux du 74 Rit de Saint-Brieuc (1914-1919) ", Mémoires de la Société d'émulation des Côtes-d'Armor, 2012, p. 439-495.

68. Arch. dép. de la Côte-d'Or, 30 U 4/54, dossier Marcelle Hellenthal : lettre de l'inculpée pour le procureur de la République, le 3 octobre 1948, écrite à la maison centrale de Rennes.

69. CARRÉ, Lily, On m'appelait la chatte..., op. cit., p. 216 et p. 274.

70. Ibidem, p. 223, à la date du 7 juillet 1947 .

71. Ibid., p. 274, en septembre 1950. 
Rarement étudié, le parcours de ces prisonnières en guerre que sont les condamnées à mort permet de mieux comprendre l'épuration, qui est à la fois une période et un processus de transition, entre crise et recomposition de la société.

Durant un temps troublé, marqué par des statuts de prisonniers extrêmement confus et poreux, la prison demeure un véritable enjeu. Passée la saison d'une captivité décidée par la Résistance et/ou la population et placée à l'interface de l'extra-légal et du légal, vient le temps du rétablissement de l'autorité républicaine. Pour une même prisonnière, l'internement administratif et la détention peuvent succéder à la captivité, ce qui confirme que l'épuration légale n'est pas exclusive de l'épuration populaire. À défaut d'être des femmes incarcérées comme les autres en raison de la nature exceptionnelle de leur engagement et de l'amendement dont elles doivent particulièrement faire montre, leur vie derrière les barreaux est comparable à celle des prisonnières de droit commun. Les relations qu'elles entretiennent avec l'extérieur sont primordiales afin d'améliorer leur quotidien. Celles-ci, notamment à travers les échanges épistolaires, démontrent que cette " tache sociale " qu'est l'épuration ne sanctionne pas seulement les condamnées : elle touche également la famille.

Au fil des mois et des années, les anciennes collaboratrices sont proportionnellement de moins en moins nombreuses dans les geôles françaises, elles-mêmes quantitativement moins importantes que dans les premiers jours de la Libération et désormais officielles. L'ordinaire reprend le dessus, y compris en prison. Sur les 142 femmes condamnées à mort dont le parcours carcéral nous est connu et en tenant compte des libérations conditionnelles, seule la moitié est encore incarcérée en 1952. Aux lendemains de la grande loi d'amnistie de 1953, le rythme des libérations s'accélère encore et dans la seconde moitié des années 1950, elles sont moins de 10 \% à être encore derrière les barreaux. 


\section{RÉSUMÉ}

La Libération est un moment spécifique dans le rapport des femmes à l'emprisonnement. Au regard des temps ordinaires, leur part au sein la population carcérale est en nette augmentation, notamment car de nombreuses collaboratrices, ou présumées telles, sont enfermées. Cette période se distingue également par la multiplicité et l'extrême porosité des statuts de prisonnières. Certaines femmes sont tour à tour captives, internées, détenues et incarcérées, témoignant ainsi du progressif retour à la légalité républicaine. En effet, passés les temps confus d'une captivité aux mains de la Résistance et de la population, les nouvelles autorités reprennent le contrôle de l'emprisonnement. À travers le cas des femmes condamnées à mort au titre de l'épuration, il s'agit donc de comprendre les différentes scansions qui rythment la sortie de guerre. Enfin, alors que la prison est un enjeu, l'analyse de la vie quotidienne des collaboratrices derrière les barreaux doit nous permettre de répondre à une question essentielle : ces prisonnières en guerre sont-elles des prisonnières comme les autres?

\section{ABSTRACT}

The Liberation was a particular moment in the relationship between women and detention. The female prison population significantly increased compared to pre-war figures due to the imprisonment of collaborators (or presumed collaborators). This period is also distinguished by the abundance and the extremely porous nature of the prisoners' status. Some were captured, committed, detained, and imprisoned, further proof of the gradual return to the proper legal and justice system. New authorities took control of imprisonment, which contrasted with the chaotic period during which the Resistance and the general population were in charge of the prisoners. By examining the cases of the women sentenced to death because of the purge, it is possible to understand the different phases which marked the end of the war. Finally, the analysis of daily life of the female collaborators in jail allows us to answer a fundamental question: were these prisoners of war different from any other prisoners? 
